\title{
E-Textile Seam Crossing with Screen Printed Circuits and Anisotropic Conductive Film ${ }^{\dagger}$
}

\author{
Mark Catchpole \\ Conductive Transfers Ltd., Barnsley S74 9LH, UK; mark@conductivetransfers.com \\ + Presented at the International Conference on the Challenges, Opportunities, Innovations and Applications \\ in Electronic Textiles (E-Textiles 2019), London, UK, 12 November 2019.
}

Published: 11 December 2019

Keywords: E-textiles; wearables; printed circuits; screen printing; smart garments; Anisotropic Conductive Film

Screen printed circuits on substrates such as thermoplastic polyurethane (TPU) have been used for many years in certain wearable applications such as heart rate monitors. These circuits often use stretchable silver ink that provides flexibility and stretchability beyond that possible with conventional etched copper flexible printed circuits (FPCs) and makes the garment more comfortable for the wearer. Conductive transfers have recently developed a new screen printing process [1] that eliminates the TPU substrate from the garment by using transfer printing. The process has now been in production since Q3 2018 and is used to manufacture circuits for a smart garment to treat urinary incontinence using electrical muscle stimulation [2]. Most garments consist of panels of fabric that are joined using seams, which presents a challenge if the circuit needs to cross a seam. A further challenge is that conventional lead-free soldering techniques operate at $250{ }^{\circ} \mathrm{C}$, which would damage printed silver tracks. Recent experiments using a technique from the electronics industry for attaching FPCs to PCBs with anisotropic conductive film (ACF) and hot bar bonding, which can be performed at temperatures as low as $130{ }^{\circ} \mathrm{C}$, have demonstrated good potential for solving this challenge. ACF contains small beads ( $10 \mathrm{um}$ ) coated in a conductive metal together with an electrically insulating adhesive such as acrylate that melts when the ACF is placed in a hot bar bonder between the electrodes to be connected under heat and pressure. The result is conductivity in the z-direction with no conductivity in the $\mathrm{x}-\mathrm{y}$ directions so that opposing pairs of electrodes are connected in a single step with only the electrodes requiring alignment. The technique was successfully used to connect a printed heater $(5-10 \mathrm{~W})$ to an off-the-shelf lithium ion battery pack located on the other side of a seam in the fleece lining of an outdoor jacket. Pairs of tracks on either side of the seam joined with a short link circuit were also pressed onto textile and oriented such that the exposed electrodes were facing. In addition, the tracks were connected using ACF to an off-the-shelf USB A plug connector that fitted the battery pack. It is anticipated that further optimization will result in a robust, washable, and costeffective technique for creating e-textiles that are comfortable to wear.

\section{References}

1. Brook, P.T.; Bungay, R.; Revitt, M. Transfer for Application to a Surface. US Patent 10,315,402, 11 June 2019.

2. Innovo. Atlantic Therapeutics, Galway, Ireland. Available online: https://www.myinnovo.com (accessed on 11 November 2019).

(C) 2019 by the authors. Licensee MDPI, Basel, Switzerland. This article is an open access article distributed under the terms and conditions of the Creative Commons Attribution (CC BY) license (http://creativecommons.org/licenses/by/4.0/). 